\title{
Electrically tunable photonic band gap structure in monodomain blue-phase liquid crystals
}

\author{
Ramesh Manda (1)', Srinivas Pagidi', Yunjin Heo', Young Jin Lim¹, MinSu Kim $\mathbb{D}^{2}$ and Seung Hee Lee
}

\begin{abstract}
Photonic band gap materials have the ability to modulate light. When they can be dynamically controlled beyond static modulation, their versatility improves and they become very useful in scientific and industrial applications. The quality of photonic band gap materials depends on the tunable wavelength range, dynamic controllability, and wavelength selectivity in response to external cues. In this paper, we demonstrate an electrically tunable photonic band gap material that covers a wide range $(241 \mathrm{~nm})$ in the visible spectrum and is based on a monodomain bluephase liquid crystal stabilized by nonmesogenic and chiral mesogenic monomers. With this approach, we can accurately tune a reflection wavelength that possesses a narrow bandwidth $(27 \mathrm{~nm})$ even under a high electric field. The switching is fully reversible owing to a relatively small hysteresis with a fast response time, and it also shows a wider viewing angle than that of cholesteric liquid crystals. We believe that the proposed material has the potential to tune color filters and bandpass filters.
\end{abstract}

\section{Introduction}

Blue-phase liquid crystals (BPLCs) are a unique photonic band gap material that exhibit selective reflection of visible light. The selective wavelength originates from the periodic arrangement of anisotropic liquid crystal molecules. Unlike fixed band gap inorganic photonic crystals, a quasicrystalline nanostructured BPLC shows dynamic tunability of the photonic band gap by an electrical stimulus. Thus, its application in electro-optic and photonic devices, such as tunable filters and lenses, lasers, sensors, gratings, and display devices, appears feasible $e^{1-3}$.

In general, BPLCs have three distinct thermodynamic phases, namely, BPI, BPII, and BPIII. BPI and BPII form body-centered cubic (BCC) and simple cubic structures, respectively, depending on how their double-twisted cylinders (DTCs) have self-assembled. BPIII forms no

\footnotetext{
Correspondence: MinSu Kim (mkim182@jhu.edu) or Seung

Hee Lee (Ish1@jbnu.ac.kr)

${ }^{1}$ Applied Materials Institute for BIN Convergence, Department of BIN

Convergence Technology and Department of Polymer-Nanoscience and

Technology, Jeonbuk National University, Jeonju, Jeonbuk 54896, Republic of

Korea

${ }^{2}$ Department of Physics and Astronomy, Johns Hopkins University, Baltimore, MD 21218, USA
}

particular structural symmetry and is commonly referred to as a blue fog ${ }^{4,5}$. The three-dimensional (3D) crystallographic structures of BPI and BPII allow us to modulate the optical frequency in all directions. Thus, it is capable of acting as a 3D photonic crystal.

The unique structural property of BPLCs reveals not only an optically isotropic nature but also the selectivity of the visible wavelength during Bragg scattering, and this useful property enables us to facilitate the design of various functional materials and devices. However, there are some hurdles to overcome, such as a limited temperature range of the phases or short-range ordered single domains, i.e., multidomain structures that appears as mosaic textures. Many reports have suggested good approaches to these issues. First, polymer stabilization (PS) or nanoparticle incorporation can extend the limited phase range of BPLCs because the dispersed materials selectively occupy the disclination lines and minimize the free energy of the system, thereby increasing the thermal stability of BPLC $^{6-10}$. Second, we can acquire a single color by engineering a narrow band gap during Bragg scattering by constructing a monodomain BPLC on a large scale ${ }^{11-15}$. Recently, several works on achieving a 


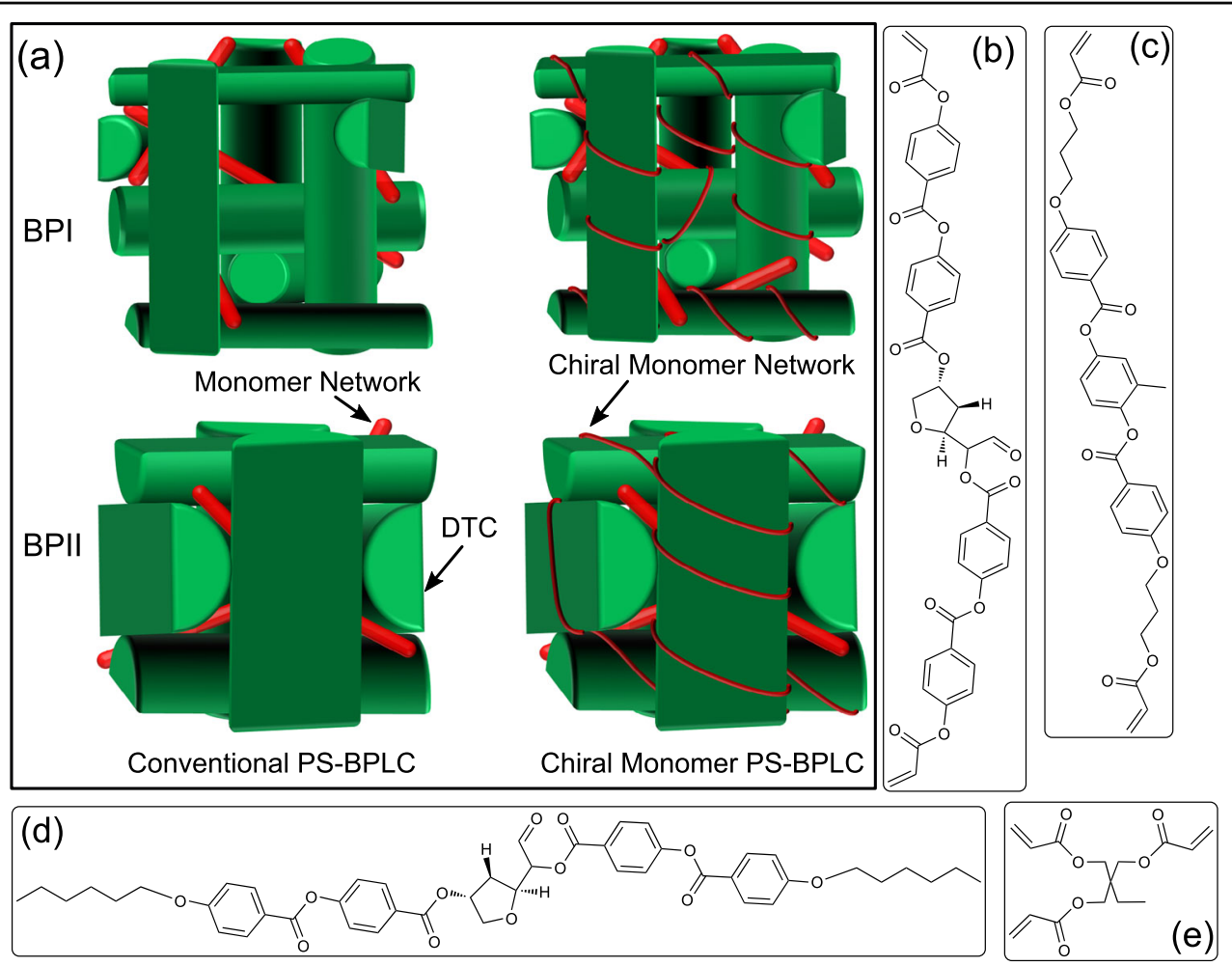

Fig. 1 PS-BPLC structure. a Schematics of a conventional PS-BPLC with polymer networks occupying disclination lines and a novel BPLC with polymer networks occupying both disclination lines and double-twist cylinders. Molecular structures of $\mathbf{b}$ chiral monomer SRM03, $\mathbf{c}$ mesogenic monomer RM257, d chiral dopant SRM17, and e monomer TMPTA.

monodomain structure in a BPLC have been reported. For instance, one can achieve it by a rubbed polyimide surface $^{13}$, a chemically patterned substrate ${ }^{16,17}$, a controlled temperature gradient ${ }^{12}$, and direct control of the structure by an electric stimulus ${ }^{15,18}$. In addition, many studies find that the use of a monodomain BPLC is the simplest approach to enhance the electro-optical performance, such as lowering the driving voltage $\mathrm{e}^{14,16,19}$.

A long-range ordered monodomain BPLC with a vivid single color can be tuned by several external stimuli. Electrical tuning is the most reasonable method among BPLC material driving schemes, such as photoinduced tuning, thermal-induced tuning or a combination of both $^{20-22}$. These approaches have shown great potential for tuning the photonic band gap of PS-BPLC. However, issues remain. For instance, the range of band gap tuning is below $100 \mathrm{~nm}^{12,20}$, beyond this, unwanted reflections due to discontinuous changes in the band gap occur ${ }^{21-24}$. The response time of field-induced tuning is slow and ranges from a few seconds to several hours, and a complicated driving scheme is sometimes required ${ }^{23}$. Thermal and electro-optic hysteresis are also commonly observed $^{21,24-27}$. Overall, dynamic control of photonic band gaps in a PS-BPLC system is still challenging, and its performance also needs to be improved.
Here, we demonstrate a monodomain PS-BPLC material whose photonic band gap is electrically tunable. The proposed monodomain PS-BPLC enables us to achieve reversible switching of the photonic band gap over a wide range of visible wavelengths with a low hysteresis. PSBPLC improvements focus on the role of reactive monomers. Before polymerization, nonmesogenic monomers prefer to occupy disclination lines to reduce the free energy, unlike other types of monomers. Thus, the polymer network is mostly generated in disclinations to hold the BPLC structure. In this work, the BPLC mixture consists of a host liquid crystal (LC), a chiral dopant, a mesogenic monomer, a nonmesogenic monomer, and a chiral monomer. Here, we emphasize that the chiral monomer plays the role of both the chiral dopant and the prepolymer. The intermolecular structure in double-twist cylinders can be firmly fixed because the chiral monomers are polymerized while retaining the chiral structure. We schematically show the internal structure of the proposed PS-BPLC with a chiral monomer in Fig. 1a. In addition to the band gap tuning by an electric stimulus, the band gap of the proposed monodomain BPLC in the ground state can be precisely tuned by controlling the concentration of chiral molecules. The proposed BPLC exhibits a narrow bandwidth of $27 \mathrm{~nm}$ and fast response time of $350 \mathrm{~ms}$, and 
we note that it shows complete recovery from the fieldinduced lattice deformation despite excessive electric field driving.

\section{Materials and methods}

The BPLC mixture employed in this study consists of a high dielectric anisotropy nematic LC (MLC2053, $\Delta \varepsilon=$ 42.6 at $1 \mathrm{kHz}, \Delta n=0.235$ at $589.3 \mathrm{~nm}$, and $T_{\mathrm{NI}}=86^{\circ} \mathrm{C}$ from Merck Advanced Technology in Korea) and a high helical twisting power chiral dopant (SRM17, HTP= $166 \mu \mathrm{m}^{-1}$ ). The phase stabilization of the BPLC was achieved by adding a chiral monomer (SRM03, HTP = $11 \mu \mathrm{m}^{-1}$ ), reactive monomer (RM257, Merck Advanced Technology, Korea), and monomer (TMPTA, trimethylolpropane triacrylate, Sigma-Aldrich). The molecular structures of the utilized chiral monomers are shown in Fig. 1b. The nematic LC to chiral dopant concentration remained constant while the chiral monomer concentration varied from 5.9 to $9.2 \mathrm{wt} . \%$. The concentrations and estimated and measured chiral pitches of the samples are listed in Table 1.

In this report, we prepared two sets of sandwiched cells to obtain multidomain and monodomain BPLCs. The substrate was deposited with indium-tin-oxide (ITO) electrodes. To achieve a monodomain BPLC, the glass substrates were coated with polyimide (AL16301, JSR Micro Korea) and unidirectionally rubbed with a velvet cloth. Thereafter, the two substrates were attached in the anti-parallel direction. No surface treatment of the substrates was performed for the multidomain BPLC samples. For both cases, the cell gap was fixed to $7 \mu \mathrm{m}$. The sample preparation was similar for both the monodomain and multidomain BPLC samples, except for the surface treatment for the monodomain samples. The complete experimental procedure is schematically illustrated in Fig. S1.

The chiral pitch $p$ of each sample mixture was measured with Grandjean-Cano lines in wedge cells. The measurement indicates that $p$ was $\sim 420,345$, and $310 \mathrm{~nm}$ for S1, S2, and S3, respectively. The BPLC was identified and characterized by polarized optical microscope (POM) (E600 POL, Nikon eclipse) connected to the camera
(DXM 1200, Nikon). The temperature of the sample was accurately maintained by a temperature controller (HSC402-STC20U, Instec) with a cooling rate of $0.3^{\circ} \mathrm{C} /$ min. Once the full BPLC appeared, the temperature was fixed, and polymerization was achieved by illumination with a $20 \mathrm{~mW} / \mathrm{cm}^{2}$ intense UV light for $10 \mathrm{~min}$ at the BPI phase $\left(\right.$ at $\left.83.6{ }^{\circ} \mathrm{C}\right)$. After polymerization, the POM characterization was performed again to find the phase extension range. After essential characterization of the BPLC phase, field-dependent characterization methods, such as photonic band gap tuning, response time measurement, and hysteresis measurement, were conducted by a photodetector (DET36A/M, Thorlabs) and an oscilloscope (DPO2024B, Tektronix) in response to a $1 \mathrm{kHz}$ square-wave voltage supplied by a function generator (AFG3101C, Tektronix) and modulated by an amplifier (A400, FLC Electronics). The Bragg reflection spectrum was measured by a reflection spectrometer (USB2000+, Ocean Optics) connected to the POM. The color values were measured for each texture observed with POM. The color values were converted to chromatic coordinates by using standardized conversion equations defined by the CIE special commission ${ }^{28}$. Here, the inbuilt $100 \mathrm{~W}$ halogen light (HLX 64623, OSRAM) in the POM was used as a light source. No other filters that were built into the POM were used. Next, we measured the viewing-angledependent reflection spectra for sample S1 at incident angles of $0^{\circ}, 15^{\circ}, 30^{\circ}$, and $70^{\circ}$ from the normal direction. The angle was produced by tilting the sample on the POM stage, and the subsequent reflection spectrum was recorded. We also examined the transmission efficiency of the S1 sample for the selective polarization of incident polychromatic light. The polarization state of the incident light was established by precisely aligning the set of optical components. The efficiency of the transmission spectra was measured by using UV-visible spectroscopy (S-3100, SCINCO). The unpolarized light from the UVvisible spectroscopy instrument was converted to circularly polarized light by using a set of polarizers and quarter-wave plates fixed appropriately. In the final step, the polymer network scaffold was observed by field emission scanning electron microscopy (FESEM) to

Table 1 The material concentrations and estimated and measured chiral pitches of the samples.

\begin{tabular}{|c|c|c|c|c|c|c|c|c|}
\hline Sample name & MLC2053 (wt.\%) & SRM17 (wt.\%) & SRM03 (wt.\%) & RM257 (wt.\%) & TMPTA (wt.\%) & $\begin{array}{l}\text { Irg-907 } \\
\text { (wt.\%) }\end{array}$ & $\begin{array}{l}\text { Estimated } \\
\text { chiral } \\
\text { pitch }^{\mathrm{a}}(\mathrm{nm})\end{array}$ & $\begin{array}{l}\text { Measured } \\
\text { chiral } \\
\text { pitch }^{\text {b }}(\mathrm{nm})\end{array}$ \\
\hline S1 & 88.7 & 1.1 & 5.2 & 3 & 1 & 1 & 417 & 420 \\
\hline S2 & 86.0 & 1.3 & 7.7 & 3 & 1 & 1 & 332 & 345 \\
\hline S3 & 85.6 & 1.4 & 8.0 & 3 & 1 & 1 & 312 & 310 \\
\hline
\end{tabular}

${ }^{a}$ Estimated by the concentration calculation based on the formula $P \sim 1 /(c \cdot H T P)$

${ }^{\mathrm{b}}$ From Fig. S4. 
estimate the polymer network formation by the chiral monomers. After completing the necessary characterization, the sample was kept in a hexane:dichloromethane (80:20) solution for $24 \mathrm{~h}$ to extract the LC and chiral dopant molecules from the sample. After confirming that the LC was completely removed, the substrates were separated by a sharp knife and used for characterization. The images were taken perpendicular to the substrate. The size of the network voids was estimated by using ImageJ, a Java-based image processing software developed by the National Institutes of Health.

\section{Results and discussion}

The fabrication process of monodomain and multidomain BPLCs is described in the Experimental section and Fig. S1. We prepared three samples that consisted of nematic LCs, a chiral dopant (SRM17), a chiral monomer (SRM03), a mesogenic monomer (RM257), and a nonmesogenic monomer (TMPTA), and their molecular structures are shown in Fig. 1c, d, e. We fabricated monodomain BPLCs by rubbing a polyimide surface which provides an appropriate roughness and anchoring condition to form a long-range monodomain BPLC, as previously reported ${ }^{19}$. We conducted photopolymerization at desired BPLC temperatures and then characterized the samples after confirming that the temperature range of the BPLCs was extended.

To determine the exact reflection wavelength and its temperature dependency, we measured the temperaturedependent Bragg reflection wavelength $\left(\lambda_{\mathrm{B}}\right)$ and investigated its corresponding texture with $\mathrm{POM}$ for the monodomain BPLC samples (Fig. 2). Unlike multidomain BPLCs that show multiple colors from mosaic textures (Fig. S2), the monodomain BPLCs show a single color (red, green, and blue) for the reflected textures over the long-range lattice arrangement (Fig. 2a-c). The Bragg reflection measurement of each monodomain sample exhibits a narrow, single reflection peak (Fig. 2d-f), whereas the multidomain BPLCs exhibit multiple peaks (Fig. S3). The measured peak wavelengths are 649, 519, and $433 \mathrm{~nm}$ for monodomain samples S1, S2, and S3, respectively, and the reflection colors of the POM images are in good agreement with the measured peak wavelengths. The measured temperature range of the BPI is fairly wide, although the BPII shows a very limited temperature range. The BPI temperatures are $20.6{ }^{\circ} \mathrm{C}, 13.1^{\circ} \mathrm{C}$, and $16.8^{\circ} \mathrm{C}$, and the temperatures for BPII are $0.4^{\circ} \mathrm{C}$, $2.4^{\circ} \mathrm{C}$, and $0.6^{\circ} \mathrm{C}$, as shown in the phase diagram of the samples in Fig. 2g. We note that the temperature dependency of the reflection wavelength is negligible (Fig. 2h). The PS method we used here leads to a strongly fixed internal structure, which retains the chiral pitch, i.e., the cubic lattice. Consequently, the thermally induced pitch change is very small such that the temperature sensitivity
$(\Delta \lambda / \Delta T)$ is calculated to be $0.11,0.42$, and $0.10 \mathrm{~nm} /{ }^{\circ} \mathrm{C}$ for $\mathrm{S} 1, \mathrm{~S} 2$, and S3, respectively. Figure 2i shows the representative Bragg reflection peaks of samples S1, S2, and S3 at $73^{\circ} \mathrm{C}, 75^{\circ} \mathrm{C}$, and $66^{\circ} \mathrm{C}$, respectively. Through this approach, we are able to tune the photonic band gap in the spectral range of $216 \mathrm{~nm}$ by adjusting the pitch of the BPLC system.

Furthermore, to gain additional insight into the influence of the chiral monomer and field-controlled tuning on the reflection wavelength, we evaluated how the Bragg reflection is determined. Theoretically, the Bragg reflection in the BPLC appears from periodically arranged crystallographic planes of the cubic lattice, expressed as $\lambda_{\mathrm{B}}=\frac{2 \bar{n} a}{\sqrt{h^{2}+k^{2}+l^{2}}}$, where $\bar{n}$ is the average refractive index of the PS-BPLC, $a$ is the lattice constant, and $h, k$, and $l$ are the Miller indices of a cubic crystal ${ }^{29}$. The value of $a$ is equal to the chiral pitch length in BPI. The selection rule for a Bragg reflection in the BCC structure is that the sum of Miller indices $(h+k+l)$ is an even number. We estimated the chiral pitch of each sample mixture by measuring the Grandjean-Cano lines in a wedge cell. The estimated pitch lengths in samples S1, S2, and S3 are $\sim 420, \sim 340$, and $\sim 310 \mathrm{~nm}$, respectively. Thus, the estimated lattice plane is (002) in S1 and S2 and (211) in S3 (Table S1 and Fig. S4). As the concentration of both the chiral dopant and chiral monomer is increased from samples S1 to S3, the chiral pitch is decreased, leading to a decrease in the cubic lattice and a shift to a shorter wavelength in the reflection peak. Furthermore, we calculated the bandwidth of each reflection peak as according to the full width at half maximum (FWHM) (measured by fitting the Gaussian equation, as shown in Fig. S5), which is $25.1,25.4$, and $26.3 \mathrm{~nm}$ for S1, S2, and S3, respectively. The obtained bandwidth is narrow, but it exhibits a relatively large temperature dependency (Fig. S6).

We examine how the Bragg wavelength changes with respect to applied electric strength for the monodomain PS-BPLC samples by observing the BPLC textures in the POM images and by analyzing the spectroscopy results. Figure 3 clearly shows that the vivid colors from the BPLC textures are in good agreement with the Bragg reflection spectra. The obtained results reveal that the reflection wavelength of the monodomain BPLC can be manipulated not only by the chiral monomer concentration but also by the electric field. When the applied electric field to sample $\mathrm{S} 1$ increases from 0 to $6.4 \mathrm{~V} / \mu \mathrm{m}$, the total tunable reflection range is $241 \mathrm{~nm}$ (Fig. 3a). The reflection wavelength is slightly shifted from 647 to $675 \mathrm{~nm}$ at a low-field strength and changes to green $(550 \mathrm{~nm})$ and blue $(433 \mathrm{~nm})$ continuously. Above $6.4 \mathrm{~V} / \mu \mathrm{m}$ and up to $7.1 \mathrm{~V} /$ $\mu \mathrm{m}$, the color disappears because the reflection wavelength reaches a range beyond the visible wavelengths. At a low-field strength, the field-induced reflection wavelength shift is caused by the reorientation of local LC 


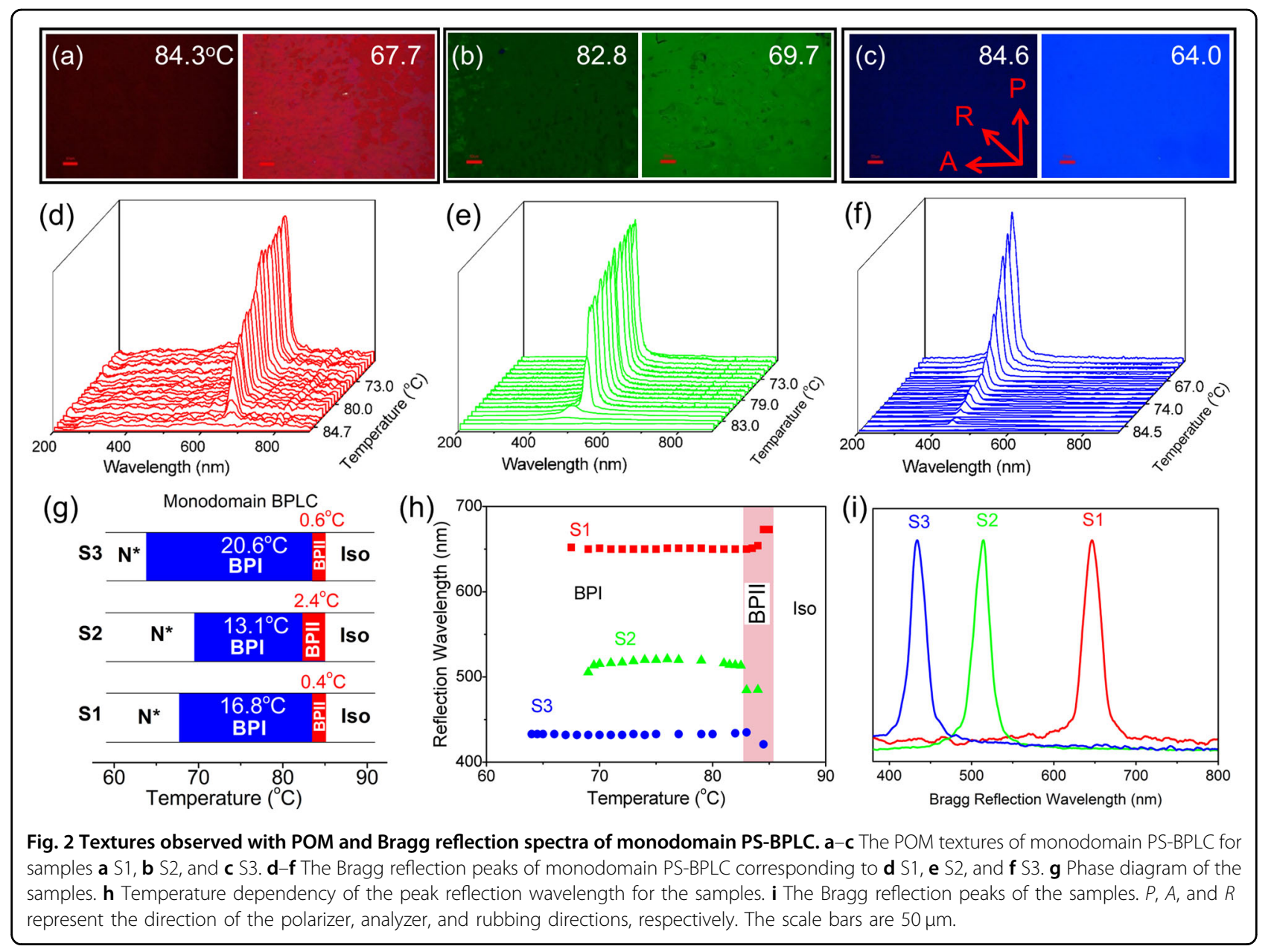

directors. As the field strength increases, the chiral pitch changes, and electrostriction occurs. On the other hand, from Fig. 3b, c, the reflection peaks from samples S2 and S3 exhibit blue and green shifts in the spectral ranges of 94 and $79 \mathrm{~nm}$, respectively. The initial reflection peaks of samples S2 and S3 appear at 517 and $434 \mathrm{~nm}$, respectively, and the peaks shift to 435 and $511 \mathrm{~nm}$ due to an applied field strength of $\sim 6.4 \mathrm{~V} / \mu \mathrm{m}$. We note that when increasing the applied field, sample $\mathrm{S} 2$ follows a similar trend to sample S1, whereas sample S3 shows opposite tendency of samples S1 and S2. The reflection peaks of S1 and S2 shift to shorter wavelengths, but they shift to longer wavelengths for S3 as the applied field increases. Such a different tendency occurs based on how the lattice planes are situated with respect to the direction of the applied electric field vector. Again, the lattice plane is estimated as (002) for samples S1 and S2, whereas it is (211) for S3. The field-induced lattice deformation of the BPLCs is determined by describing the dependence of the deformation tensor $\varepsilon_{i j}$ based on the electrostriction tensor $\gamma_{i j k l}$ on the quadratic electric field strength. Thus, $\varepsilon_{i j}=$ $\gamma_{i j k l} E_{k} E_{l}$, where the free energy minimization gives rise to three independent electrostriction coefficients $\gamma_{i i i i}, \gamma_{i i j j}$, and $\gamma_{i j i j}$ for cubic symmetry ${ }^{30}$. The sign of the $\gamma$ in the BPI with a positive dielectric anisotropy LC is determined in reference ${ }^{30}$; that is, $\gamma_{i i i i}$ is negative, $\gamma_{i i j j}$ is positive, and $\gamma_{i j i j}$ is positive. Thus, for the (002) plane, when the electric field direction parallel is to the fourfold axis (001), the lattice dimension along the field decreases as the field strength increases to satisfy the negative sign of $\gamma_{i i i i}$. For the (211) plane in S3, the tendency is opposite to that for S1 and S2 owing to the positive sign of $\gamma_{i j i j}$, i.e., the lattice constant decreases. A schematic of the lattice distortion against the applied field is shown in Fig. 4. The roughness of the surface depends on the parameters for the rubbing method, for instance, the rotation speed, the depth of contact, and the length of the fibers on the roller. The surface roughness is the result of the BPLC lattices being located along the (200) plane for S1 and S2, but the shortest chiral pitch that occurred for S3 is preferred to locate along the (211) plane. For sample S1, we achieve band gap tuning over a wide spectral range from red to blue, which is two times wider than previously reported results $^{11,12}$. 


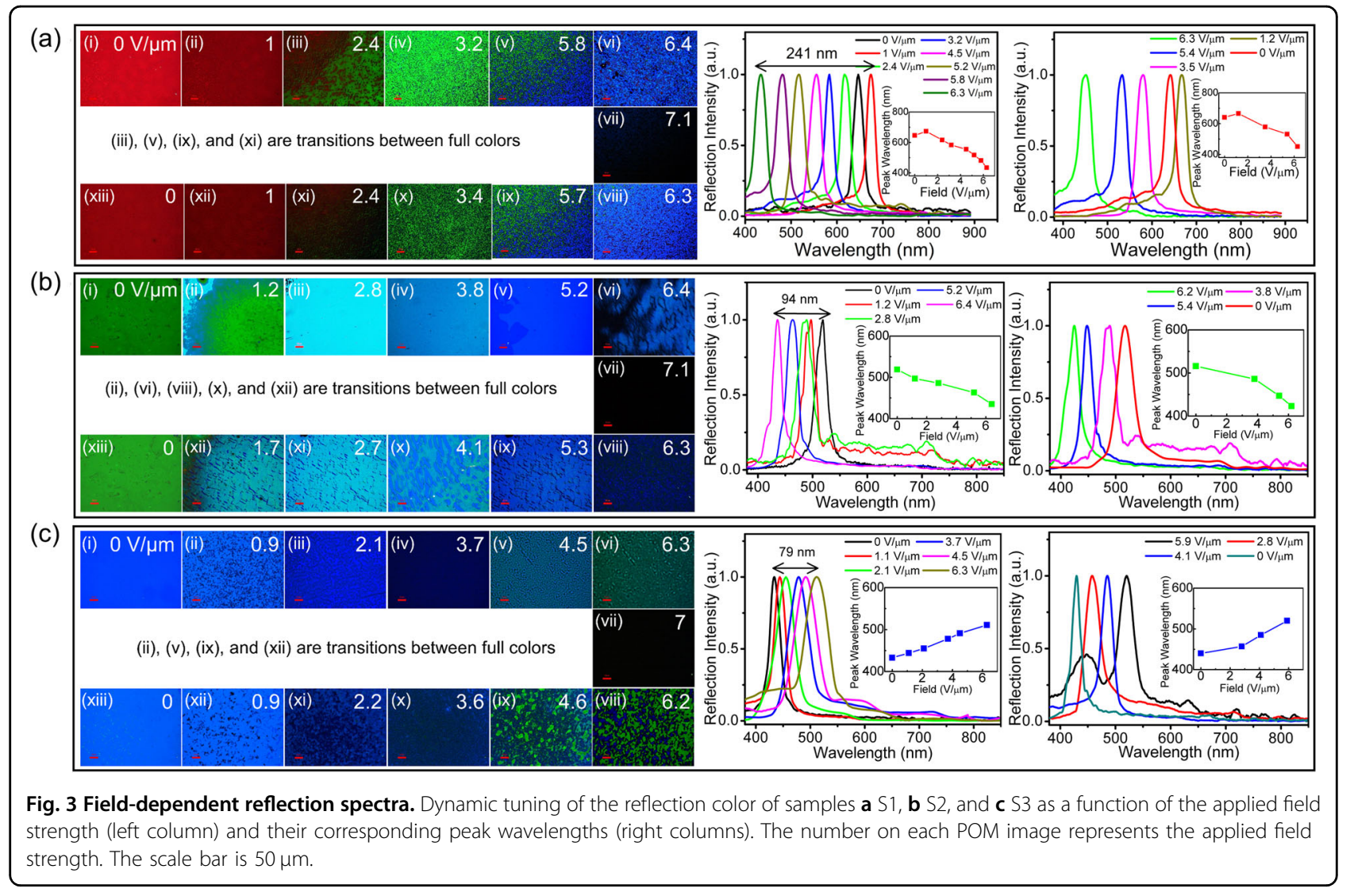

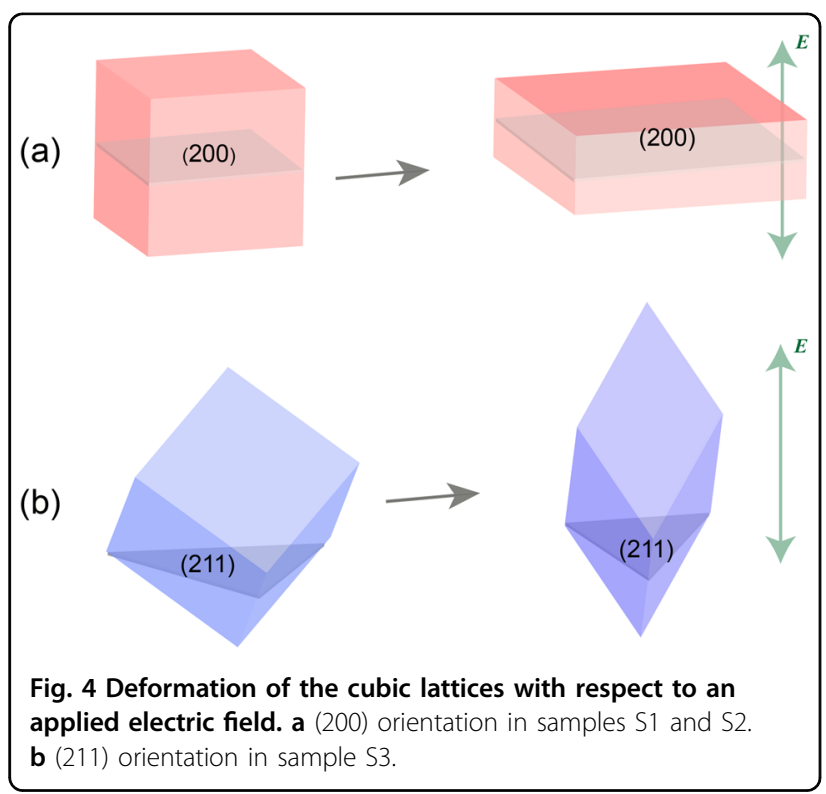

The main role of the nonmesogenic monomer after polymerization is to maintain the phase and to prevent the field-induced phase transition because the part of the polymer network that comprises the nonmesogenic monomer mostly occupies the disclination lines. The mesogenic monomer is polymerized to interconnect the LCs and enhances the thermodynamic stability, but this polymer network does not form a chiral structure upon the response of the electric field. Thus, a high concentration of achiral monomers results in a local reorientation. However, the other part of the polymer network that comprises the chiral monomer maintains the DTC structures, i.e., the BPLC lattices, so that it prefers to induce electrostriction rather than a local reorientation. One may find the optimum balance between chiral and achiral monomer concentrations to achieve both a high stability and tunability by understanding this relation.

The polymer stabilization in monodomain BPLCs guarantees long-term stability during operation (more than 3 months). Moreover, the uniformity of the texture color is improved after the removal of the electric field. This result suggests that we can improve the spatial organization of the cubic lattice by an electric field. The great advantage of the system is that the electrically tunable reflection wavelengths are consistently repeatable for a number of field cycles.

We calculated the hysteresis upon band gap switching in which the voltage ramps up and down, as shown in Fig. $5 \mathrm{a}$. In the PS-BPLC, it is known that an incomplete or slow relaxation from the lattice deformation after voltageoff causes hysteresis ${ }^{31,32}$. Here, we define the band gap 


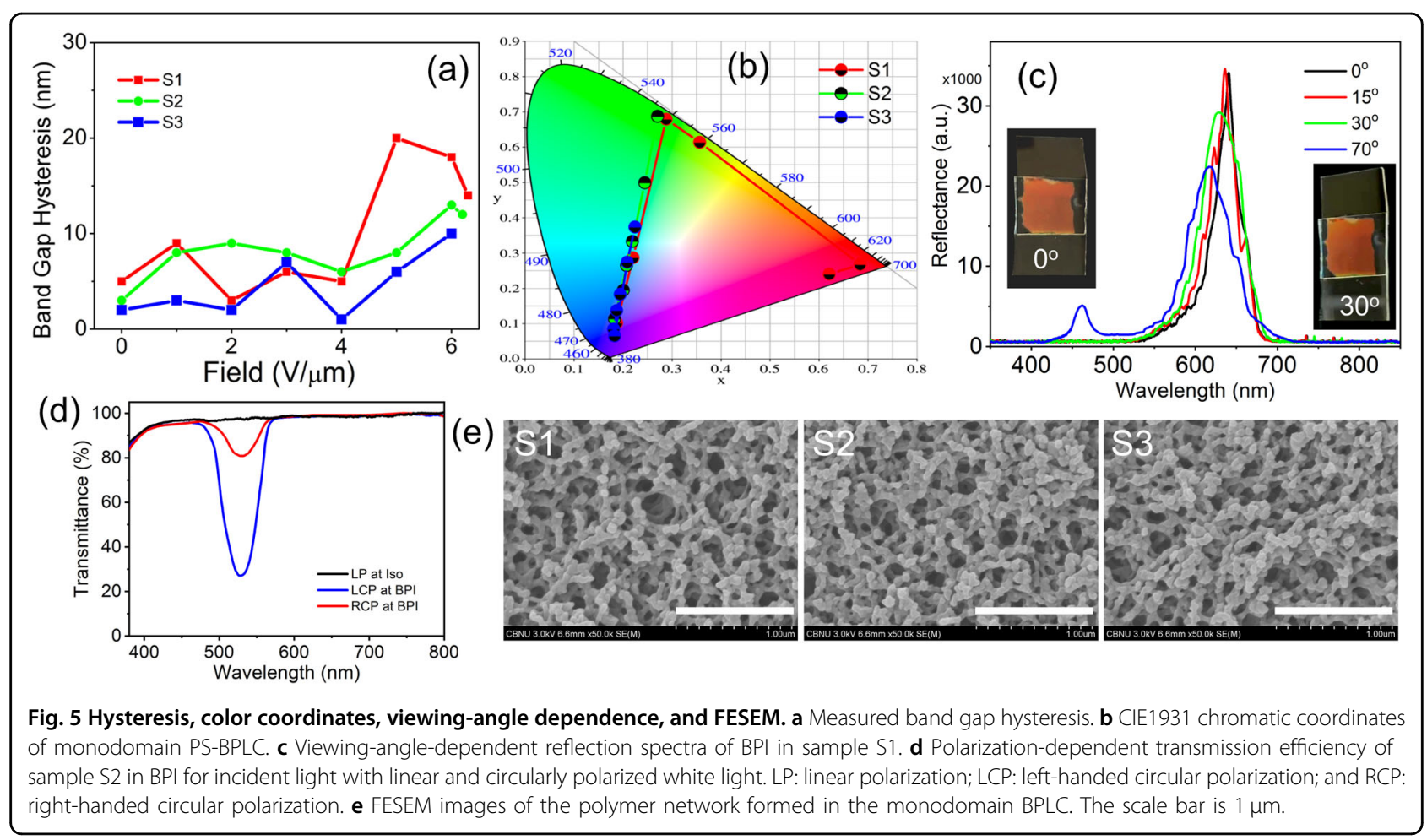

hysteresis $\Delta H=\lambda_{\mathrm{B} 1}-\lambda_{\mathrm{B} 2}$, where $\lambda_{\mathrm{B} 1}$ and $\lambda_{\mathrm{B} 2}$ are the reflection peak wavelengths related to the field increase and decrease, respectively, at a particular field during sweeps. The measured $\Delta H$ is smaller than $10 \mathrm{~nm}$ for samples S2 and S3, while it is smaller than $20 \mathrm{~nm}$ for S1. This result implies that the lattice distortion quickly and fully recovers back to its original structure owing to the polymerization of the internal structure and disclination. Although the PS-BPLC has the potential for negligible hysteresis, a finite hysteresis is obtained from the electrostriction effect ${ }^{33,34}$. We also verify the bandwidth at each peak with respect to the applied electric fields over the samples. The measured bandwidth of samples S1, S2, S3 has an average of 28.7, 27.3, and $40 \mathrm{~nm}$, respectively, when ramping up the electric field and 32.5, 30.5, and $29.2 \mathrm{~nm}$, respectively, when ramping down the electric field. Each measurement is shown in Fig. S7.

To evaluate the color properties of the BPLC, we measured the chromaticity of the prepared monodomain samples based on the International Commission on Illumination (CIE) 1931 color space chromaticity diagram with respect to applied electric fields (Fig. 5b). The detailed calculations are shown in Tables S2-S4. The chromaticity coordinates of sample S1 range from red to blue colors: red $(0.62,0.24)$ at $0 \mathrm{~V} / \mu \mathrm{m}$, green $(0.28,0.68)$ at $3.2 \mathrm{~V} / \mu \mathrm{m}$, and blue $(0.18,0.10)$ at $6.4 \mathrm{~V} / \mu \mathrm{m}$. Sample S2 ranges from green to blue colors: green $(0.27,0.69)$ at $0 \mathrm{~V} /$ $\mu \mathrm{m}$ and blue $(0.20,0.19)$ at $6.4 \mathrm{~V} / \mu \mathrm{m}$. Sample S3 ranges from blue to green: blue $(0.18,0.14)$ at $0 \mathrm{~V} / \mu \mathrm{m}$ and green
$(0.22,0.37)$ at $6.3 \mathrm{~V} / \mu \mathrm{m}$. Sample S1 shows highly saturated red, blue, and green color coordinates in the color space, exhibiting a color gamut of $85 \%$ of the NTSC (National Television System Committee) color gamut.

We also measured viewing-angle-dependent reflection spectra for sample $\mathrm{S} 1$ at incident angles of $0^{\circ}, 15^{\circ}, 30^{\circ}$, and $70^{\circ}$ from the normal direction. The Bragg reflection spectrum shows that as the incident angle increases, the peak wavelength shifts toward shorter wavelengths; there are slight extensions of the bandwidth and a decrease in the intensity. The cubic lattice closely resembles and homogeneously grows with matching symmetric axes in the monodomain BPLC. At this position, a small alteration in the lattice resembling a longer scale causes a slight change in the bandwidth and reflection wavelength shift. At the incident angle of $70^{\circ}$, a relatively weak peak in the blue region for $\lambda_{\mathrm{B}} \sim 460 \mathrm{~nm}$ appears. This peak originates from another plane of the Bragg lattice. Despite the appearance of another color, the sample exhibits a much wider viewing angle property than the cholesteric phase of this sample shown in Fig. S8. The photographic images in the insets of Fig. $5 \mathrm{c}$ are in good correlation with the spectra measurements, proving that the proposed BPLC works as an excellent photonic device not only in the normal direction but also at wide viewing angles.

To evaluate the electro-optic performance of the possible applications, we measured the response time of the samples (Fig. S9). The rising time is defined as the time required to switch from the initial lattice to the deformed 
lattice structure upon electrostriction, and the decay time is defined the reverse process. The measured results for samples S1, S2, and S3 show rising times of 180, 120, and $150 \mathrm{~ms}$ and decaying times of 170,150 , and $110 \mathrm{~ms}$, respectively. A few hundred $\mathrm{ms}$ response time is not as fast as the response time of the local reorientation in the BPLC $^{35,36}$. Nevertheless, the achieved switching times are faster than the earlier reports on the electrostriction effect $^{20-22}$.

We examine how efficiently circularly polarized light is transmitted depending on the handedness of the light when traveling through the monodomain PS-BPLC. Here, the incident light is first converted to linearly polarized light by a polarizer and then converted to circularly polarized light by placing a quarter-wave plate with an angle of $45^{\circ}$ or $-45^{\circ}$ to the linear polarization direction for left-handed or right-handed circular polarization (LCP or $\mathrm{RCP}$, respectively). This optical setup enables us to easily switch between two polarization states. We quantified the transmittance by comparing the measured optical intensity for BPLCs with both a LCP and RCP to the isotropic phase (above $\mathrm{T}_{\mathrm{NI}}$ ) with the LP. As shown in Fig. 5d, we achieve $74 \%$ and $19 \%$ transmission efficiency for the LCP and RCP at $528 \mathrm{~nm}$, respectively. The result reveals that an increased efficiency is achieved for the LCP owing to the same handedness of the chiral dopant as the incident light. This property can be used as a bandpass filter for circularly polarized light.

Finally, we observed the polymer network directly by using FESEM. As shown in Fig. 5e, a network in the polymer matrix is achieved, in which a slight alteration in the architecture of the network from samples S1 to S3 is observed such that the average void size in the network is measured to be 210,180 , and $160 \mathrm{~nm}$ for samples S1, S2, and S3, respectively. It should be noted that the voids in the polymer network in our samples is smaller than that in the previous literature reports ${ }^{3,8,13,37}$. In addition, the density of the polymer network increases from S1 to S3 due to an increase in polymer network formation inside the DTCs associated with an increasing chiral monomer concentration. The chiral monomer-stabilized monodomain BPLC introduced in this work has the potential to improve the color gamut in LCDs by cutting a specific wavelength to reduce color mixing associated with the intrinsic property of color filters ${ }^{38,39}$. Reversible switching with a fast response will be another benefit for this device, which opens new prospects for it. Considering the temperature range of the phase, the device can be used either where the temperature is not a critical issue or for thermal imaging devices.

\section{Conclusion}

We demonstrated dynamic control of the photonic band gap in monodomain polymer-stabilized blue-phase liquid crystals via an electric stimulus. The polymer network in the proposed system is formed in not only disclination lines but also bulk liquid crystals, such that it strongly holds the internal director field and chirality and maintains the internal structure of the system even after a strong lattice deformation by the electrostriction effect. By controlling the concentration of the chiral monomer and the ratio of LC to chiral dopant in the BPLC, we are able to set the color of the photonic effect with its controllable spectral range of $\sim 216 \mathrm{~nm}$. By adjusting the electric field strength, the photonic band gap over the $241 \mathrm{~nm}$ range can be tuned with a fast switching time of $<350 \mathrm{~ms}$. In addition, the tunable band gap covers a wide color space ( $85 \%$ of NTSC color gamut) with high color purity owing to the narrow bandwidth of $31 \mathrm{~nm}$. Moreover, the measured efficiency of the reflectivity is $74 \%$ for circularly polarized light. Importantly, the band gap switching caused by the electrostriction effect is completely reversible and has a decreased hysteresis. The single, narrow reflection peak with wide-range color tunability is promising for use as tunable color filters. The wide band gap tunability with a fast response time and negligible hysteresis makes this a promising approach for novel photonic applications.

\section{Acknowledgements \\ This research was supported by the Basic Science Research Program through the National Research Foundation of Korea (NRF) funded by the Ministry of Education (2016R1D1A1B01007189) and by the National Research Foundation of Korea (NRF) grant funded by the Korean government (MSIT) (No. 2019R1A5A8080326).}

\section{Conflict of interest}

The authors declare that they have no conflict of interest.

\section{Publisher's note}

Springer Nature remains neutral with regard to jurisdictional claims in published maps and institutional affiliations.

Supplementary information is available for this paper at https://doi.org/ 10.1038/s41427-020-0225-8.

Received: 28 December 2019 Revised: 1 March 2020 Accepted: 11 March 2020.

Published online: 29 May 2020

\footnotetext{
References

1. Cao, W., Munoz, A., Palffy-Muhoray, P. \& Taheri, B. Lasing in a threedimensional photonic crystal of the liquid crystal blue phase II. Nat. Mater. 1, 111 (2002).

2. Li, Y. \& Wu, S. T. Polarization independent adaptive microlens with a bluephase liquid crystal. Opt. Express 19, 8045-8050 (2011).

3. Manda, R. et al. Ultra-fast switching blue phase liquid crystals diffraction grating stabilized by chiral monomer. J. Phys. D. Appl. Phys. 51, 185103 (2018).

4. Crooker, P. P. Plenary Lecture. The blue phases. A review of experiments. Liq. Cryst. 5, 751-775 (1989).

5. Kitzerow, H. S. Blue phases: prior art, potential polar effects, challenges. Ferroelectrics $395,66-85$ (2010).

6. Kikuchi, H., Yokota, M., Hisakado, Y., Yang, H. \& Kajiyama, T. Polymer-stabilized liquid crystal blue phases. Nat. Mater. 1, 64 (2002).

7. Wang, L. et al. Polymer-stabilized nanoparticle-enriched blue phase liquid crystals. J. Mater. Chem. C. 1, 6526-6531 (2013).
} 
8. Kim, M. S. et al. Reduced graphene oxide (RGO) enriched polymer network for highly-enhanced electro-optic performance of a liquid crystalline blue phase. RSC Adv. 7, 16650-16654 (2017).

9. Kasch, N., Dierking, I. \& Turner, M. Stabilization of the liquid crystalline blue phase by the addition of short-chain polystyrene. Soft Matter 9, 4789-4793 (2013).

10. Kemiklioglu, E., Hwang, J. Y. \& Chien, L. C. Stabilization of cholesteric blue phases using polymerized nanoparticles. Phy. Rev. E 89, 042502 (2014)

11. Sridurai, V., Mathews, M., Yelamaggad, C. V. \& Nair, G. G. Electrically tunable soft photonic gel formed by blue phase liquid Crystal for switchable colorreflecting mirror. ACS Appl. Mater. Inter. 9, 39569-39575 (2017).

12. Chen, C. W. et al. Large three-dimensional photonic crystals based on monocrystalline liquid crystal blue phases. Nat. Commun. 8, 727 (2017).

13. Jo, S. Y. et al. Polymer stabilization of liquid-crystal blue phase ॥ toward photonic crystals. ACS Appl. Mater. Inter. 9, 8941-8947 (2017).

14. Oton, E., Netter, E., Nakano, T. \& Inoue, F. Monodomain blue phase liquid crystal layers for phase modulation. Sci. Rep. 7, 44575 (2017).

15. Chen, Y. \& Wu, S. T. Electric field-induced monodomain blue phase liquid crystals. Appl. Phys. Lett. 102, 171110 (2013).

16. Martínez-González, J. A. et al. Directed self-assembly of liquid crystalline bluephases into ideal single-crystals. Nat. Commun. 8, 15854 (2017).

17. Li, X. et al. Mesoscale martensitic transformation in single crystals of topological defects. Proc. Nat. Acad. Sci. USA 114, 10011-10016 (2017).

18. Yan, J., Lin, J., Li, Q. \& Li, R. Z. Influence of long-lasting electric field on the formation of monodomain polymer stabilized blue phase liquid crystals. J. Appl. Phys. 125, 024501 (2019).

19. Nayek, P. et al. Tailoring monodomain in blue phase liquid crystal by surface pinning effect. Appl. Phys. Express 5, 051701 (2012)

20. Lin, J. D., Wang, T. Y., Mo, T. S., Huang, S. Y. \& Lee, C. R. Wide-band spatially tunable photonic bandgap in visible spectral range and laser based on a polymer stabilized blue phase. Sci. Rep. 6, 30407 (2016).

21. Chen, C. W. et al. Electric field-driven shifting and expansion of photonic band gaps in 3D liquid photonic crystals. ACS Photonics 2, 1524-1531 (2015).

22. Liu, H. Y., Wang, C. T., Hsu, C. Y., Lin, T. H. \& Liu, J. H. Optically tuneable blue phase photonic band gaps. Appl. Phys. Lett. 96, 121103 (2010).

23. Lin, T. H. et al. Red, green and blue reflections enabled in an optically tunable self-organized 3D cubic nanostructured thin film. Adv. Mater. 25, 5050-5054 (2013).
24. Lu, S. Y. \& Chien, L. C. Electrically switched color with polymer-stabilized bluephase liquid crystals. Opt. Lett. 35, 562-564 (2010).

25. Liang, $X$. et al. Effects of polymer micro-structures on the thermo-optical properties of a flexible soft-mater film based on liquid crystals/polymer composite. Polymer 146, 161-168 (2018).

26. Du, X. W. et al. Symmetric continuously tunable photonic bandgaps in bluephase liquid crystals switched by an alternating current field. ACS Appl. Mater. Inter. 11, 22015-22020 (2019).

27. Manda, R. et al. Phase stabilisation of blue-phase liquid crystals using a polymerisable chiral additive. Liq. Cryst. 44, 1059-1068 (2017).

28. Fairman, H. S., Brill, M. H. \& Hemmendinger, H. How the CIE 1931 colormatching functions were derived from Wright-Guild data. Color Res. Appl. 22 11-23 (1997).

29. Kikuchi, H. in Liquid Crystalline Functional Assemblies and Their Supramolecular Structures, 99-117 (Springer, Berlin, Heidelberg, 2007).

30. Heppke, G., Jerome, B., Kitzerow, H. S. \& Pieranski, P. Electrostriction of the cholesteric blue phases BPI and BPII in mixtures with positive dielectric anisotropy. J. de. Phys. 50, 2991-2998 (1989).

31. Lan, Y. F., Tsai, C. Y., Lu, J. K. \& Sugiura, N. Mechanism of hysteresis in polymernetwork stabilized blue phase liquid crystal. Polymer 54, 1876-1879 (2013).

32. Kim, M. S. \& Chien, L. C. Topology-mediated electro-optical behaviour of a wide-temperature liquid crystalline amorphous blue phase. Soft Matter 11 8013-8018 (2015)

33. Rao, L. et al. Critical field for a hysteresis-free BPLC device. J. Disp. Technol. 7 627-629 (2011).

34. Liu, Y. et al. A hysteresis-free polymer-stabilised blue-phase liquid crystal. Liq. Cryst. 41, 1339-1344 (2014).

35. Choi, H., Higuchi, H. \& Kikuchi, H. Fast electro-optic switching in liquid crystal blue phase II. Appl. Phys. Lett. 98, 131905 (2011).

36. Choi, H., Higuchi, H. \& Kikuchi, H. Electrooptic response of liquid crystalline blue phases with different chiral pitches. Soft Matter 7, 4252-4256 (2011).

37. Gandhi, S. S., Kim, M. S., Hwang, J. Y. \& Chien, L. C. Electro-optical memory of a nanoengineered amorphous blue-phase-III polymer scaffold. Adv. Mater. $\mathbf{2 8}$ 8998-9005 (2016)

38. Wyatt, D., Chen, H. \& Wu, S. T. Wide-color-gamut LCDs with vivid color LED technology. SID Symp. Dig. Tech. Pap. 48, 992-995 (2017).

39. Chen, $\mathrm{H}$. et al. Enlarging the color gamut of liquid crystal displays with a functional reflective polarizer. Opt. Express 25, 102-111 (2017). 